\title{
$\triangle$ Original contribution \\ Transurethral microwave hyperthermia in the treatment of chronic nonbacterial prostatitis
}

\author{
MATTHEW P. MENÉ, DO, MSc; PHILLIP C. GINSBERG, DO, JD, MSc; \\ LEONARD H. FINKELSTEIN, DO, MSc; SAMUEL J. MANFREY, DO, MSc; \\ LAURENCE BELKOFF, DO, MSc; FRANCIS OGBOLU, DO; \\ DAWN OSBORNE, RNC, BSN
}

Chronic nonbacterial prostatitis is an ill-understood and difficult-to-diagnose disease. Symptoms of chronic nonbacterial prostatitis are similar to those of chronic prostatitis and include low back pain, frequency, dysuria, perineal discomfort, and painful ejaculation. In view of uncertainty about etiology, treatment of chronic nonbacterial prostatitis remains speculative. Most treatment is aimed at relieving symptoms and not at curing the disease. Because of the troublesome nature of chronic nonbacterial prostatitis and the poor results obtained from traditional treatment methods, a new modality of transurethral microwave hyperthermia was investigated. Six patients were treated from January 1994 through June 1995 by use of transurethral microwave hyperthermia. These men were treated four times during a 2-week period. Their average symptom score decrease was $74.9 \%$ and was associated with minimal morbidity. Based on this result, it is concluded that transurethral microwave hyperthermia is a safe and effective treatment modality for chronic nonbacterial prostatitis.

(Key words: Prostatitis, microwave hyperthermia)

$\mathrm{C}$ hronic nonbacterial prostatitis is an ill-understood disease that is difficult to diagnose. Its symptoms are similar to those of chronic prostatitis and include low back pain, frequency, dysuria, perineal discomfort, and painful ejaculation. No certainty exists as to the cause of chronic nonbacterial prostatitis; infectious disease, autoimmunity, and even allergy have been speculated to be the cause. In view of the uncertainty about etiology, treatment of nonbacterial prostatitis remains a problem. Most treatment is aimed at relieving symptoms and not at curing the disease. Because of

From the Division of Urology, Philadelphia College of Osteopathic Medicine, Philadelphia, Pa.

Correspondence to Leonard $\mathrm{H}$. Finkelstein, DO, MSc, Division of Urology, Philadelphia College of Osteopathic Medicine, 4170 City Ave, Philadelphia, PA $19131-1694$. the troublesome nature of chronic nonbacterial prostatitis and the poor results obtained from all traditional modes of treatment, a new treatment modality of transurethral microwave hyperthermia was investigated.

The sensitivity of cancer cells to elevated temperature $\left(>42^{\circ} \mathrm{C}\right)$ is well established and documented. ${ }^{1}$ The therapeutic effects of hyperthermia on various solid tumors has been known for more than a century, and during the past decade, there has been increasing use of clinical local hyperthermia alone or combined with other established modalities in the treatment of malignant and benign disease. ${ }^{2}$ Local hyperthermia is achieved by means of regional perfusion of the extremities, hot air, water bath immersion, ultrasound, radiofrequency, and microwaves.
Delivery of hyperthermia to the prostate, for the treatment of benign prostatic hyperplasia, by use of a transurethral probe, was studied at Akita University School of Medicine in Akita, Japan. ${ }^{3}$ The results of these preliminary studies and animal experiments indicate that heat coagulation of the prostate by microwave radiation causes necrosis with minimal inflammatory reaction, and that the size of the thermal reaction in the prostate is regulated by the length of the antenna, output intensity, or duration of the microwave exposure. 4 The results also indicate that this new method should be a safe procedure because microwaves penetrate only several centimeters into biologic tissue owing to the fact that microwave energy is absorbed into tissue exponentially relative to tissue depth. 4,5

The safety of microwave hyperthermia to the prostate has been proved in earlier studies and clinical trials in support of Food and Drug Administration premarket approval. In experiments in which local deep microwave hyperthermia was applied to the prostate of dogs and of rabbits, it was demonstrated by repeated, sequential microscopic examinations that heating to temperatures up to $42^{\circ} \mathrm{C}\left( \pm 0.5^{\circ} \mathrm{C}\right)$ for 90 minutes is safe and may be repeated with no evidence of deep injury to the prostate. ${ }^{5}$

Transurethral microwave hyperthermia has been used for various prostatic diseases including benign prostatic hyperplasia $(\mathrm{BPH}) .2,6$ The initial results reported in the studies were encouraging. Transurethral microwave hyperthermia to the prostate is generally thought to be a safe and effective treatment modality for various diseases of the prostate, including $\mathrm{BPH}$ and prostate carcinoma. ${ }^{7}$

Because of the troublesome nature of chronic nonbacterial prostatitis and the poor results obtained in traditional methods of treatment, the new treatment modality of transurethral microwave hyperthermia is undergoing investigation. Our hypothesis is that transurethral microwave hyperthermia with its resultant decrease in overall prostatic size and ablation of inflamed prostatic tissue will greatly enhance the qual- 
ity of life in these patients. By decreasing the volume of the inflamed prostate, subjective complaints of prostatic discomfort are expected to decrease as well. The objective of this study was to evaluate the safety and efficacy of microwave hyperthermia in the treatment of chronic nonbacterial prostatitis.

\section{Materials and methods}

In each treatment, a temperature of at least $48^{\circ} \mathrm{C}\left( \pm 1^{\circ} \mathrm{C}\right)$ was applied to the prostate via a specially designed Foley catheter for 60 minutes. Patient eligibility included men 25 to 50 years of age with documented chronic nonbacterial prostatitis. Chronic nonbacterial prostatitis was documented by appropriate subjective complaints (see introductory text), and includes a negative expressed prostatic secretion and empiric treatment for Ureaplasma and Chlamydia infections. Patients did not receive other hyperthermia for 4 weeks before treatment. Patients had no previous prostate surgery and were all competent to give informed consent. Excluded were men with prostate carcinoma, acute bacterial prostatitis, acute urinary tract infection, anal or rectal disease, cardiac pacemakers, and metallic implants in the pelvic region.

All patients underwent a general pretreatment evaluation comprising a detailed genitourinary history; complete blood cell count; levels of prostate-specific antigen (PSA), electrolytes, blood urea nitrogen, creatinine, and urinalysis; urine culture; as well as a physical examination, including a rectal examination. Further urologic pretreatment evaluation included a transrectal ultrasound study, uroflow, measurement of postvoid residual volume, cystoscopy, cystometrogram, and semen analysis. The purpose of this extensive pretreatment evaluation was to eliminate other possible causes of symptoms, such as bladder carcinoma, prostate carcinoma, $\mathrm{BPH}$, urinary tract infection, or neurogenic bladder. All men completed a modified American Urological Association (AUA) symptom score index pretreatment and posttreatment (Table 1). The scores ranged from 0 to 60 and pro- vided the subjective basis for treatment success.

Each patient received four 60 -minute hyperthermia treatments during a 2week period. Hyperthermia was administered using the ProsTec 3000 microwave equipment with interstitial antennas inside a specially designed Foley catheter (Thermal Therapeutics, Dallas, Tex). Temperature was recorded using a flexible fiberoptic sensor centered in the catheter. A four-point linear array with distal tip inserted to the base of the balloon and others placed at $1-\mathrm{cm}$ intervals measured the intracatheter temperatures. Temperatures were monitored continuously using the computer-controlled recording and display system. The ProsTec 3000 automatically displays and records all treatment parameters including heat-up, plateau and washout, maximum heat achieved, time remaining, elapsed time, time above a given temperature, and thermal dose. All patients achieved a temperature of $48^{\circ} \mathrm{C}\left( \pm 1^{\circ} \mathrm{C}\right)$ for 60 minutes.

Patients were evaluated at 1, 3, 6, 9, and 12 months posttreatment (Table 2). Modified AUA symptom scoring was done at each evaluation to monitor subjective response to treatment. This modified symptom score was the basis for assessing success, as chronic nonbacterial prostatitis often must be evaluated subjectively. Unfortunately, no reliable objective criteria exist to document response in this disease. Placebo effect was considered and has been documented in other studies to be approximately $30 \%$. We therefore were expecting results greater than $30 \%$ to rule out placebo effect.

\section{Results}

Six men were treated during the interval from January 1994 to June 1995 . They ranged in age from 28 to 47 years (mean, 37.3 years). All men had chronic nonbacterial prostatitis, documented by a negative expressed prostatic secretion, specific subjective complaints, and empiric treatment for Chlamydia and Ureaplasma infections. All men underwent the prescribed pretreatment evaluation, and no overt genitourinary abnormalities were noted. The men were followed up posttreatment from 6 to 16 months to date.

Four of the six men underwent the prescribed four treatments. One man underwent only one treatment and voluntarily withdrew from the study, and one patient was dropped from the study after a vasovagal response during his first catheter insertion.

Among the four patients treated for the full prescribed course of transurethral microwave hyperthermia, the mean symptom score decrease was $74.9 \%$ (range, $72.0 \%$ to $78.9 \%$ ) with a standard deviation of $\pm 3.53(P<.001)$ using the Student's $t$ test (Table 3). Even the patient treated only once had a symptom score decrease of $47.4 \%$, which is better than expected placebo effects.

Five of the six men underwent posttreatment evaluation, and there were no significant laboratory changes from the pretreatment evaluation. Two of the six $(33.3 \%)$ men complained of dysuria and edema of the meatus after treatment. These complaints were thought to be secondary to the heat generated by the microwave hyperthermia and resolved spontaneously without sequelae. Of the six patients, one $(16.6 \%)$ had a mild meatal stenosis pretreatment and complained of hematuria posttreatment which was self-limited and thought to be the result of the meatal stenosis. Three $(50.0 \%)$ of the six patients complained of a symptom of urethritis which was transient and resolved 24 hours after treatment.

\section{Discussion}

There are two general categories of chronic prostatitis: bacterial and nonbacterial. These two diseases have the same symptoms, but they differ in diagnosis and treatment. Low back pain, perineal discomfort, dysuria, urgency, frequency, and painful ejaculation are some of the symptoms.6,8-11 Chronic bacterial prostatitis can be diagnosed by a positive expressed prostatic secretion and treated with long-term antibiotic therapy, up to 3 months. Chronic nonbacterial prostatitis is ill understood, difficult to diagnose, and often unrespon- 
Table 1

Urinary Symptoms (Symptom Score Criteria)-

American Urological Association Symptom Index *

Question

$\begin{gathered}\text { Not at } \\ \text { all }\end{gathered}$
$\begin{gathered}<1 \text { Time } \\ \text { in } 5\end{gathered}$ the time

About half

$>$ Half

Almost

the time

the time

always

1. Over the past month or so, how often have you had a sensation of not emptying your bladder completely after you finished urinating?

0

0

2 hours after you finished urinating?

3. Over the past month or so, how often have you found you stopped and started again several times when you urinated?

4. Over the past month or so, how often have you found it difficult to postpone urination?

5. Over the past month or so, how often have you had a weak urinary stream?

6. Over the past month or so, how often have you had to push or strain to begin urination?

7. Over the past month or so, how often have you experienced pain or burning while urinating?

0

0

1

2

3

4

5

8. Over the past month or so, how often have you experienced low back pain?

9. Over the past month or so, how often have you experienced perineal discomfort?

0

1

2

3

4

10. Over the past month or so, how often

have you experienced low abdominal pain?

0

1

2

3

4

11. Over the past month or so, how often have you experienced pain with ejaculation?

0

1

2

3

4 5

12. Over the past month or so, how many times did you most typically get up to urinate from the time you went to bed at night until the time you got up in the morning?

Number of times

Symptom score

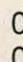

$0 \quad 1$

1
1

2

3

3
5

5

5

5 


\section{Table 2}

Data Collection Schedule

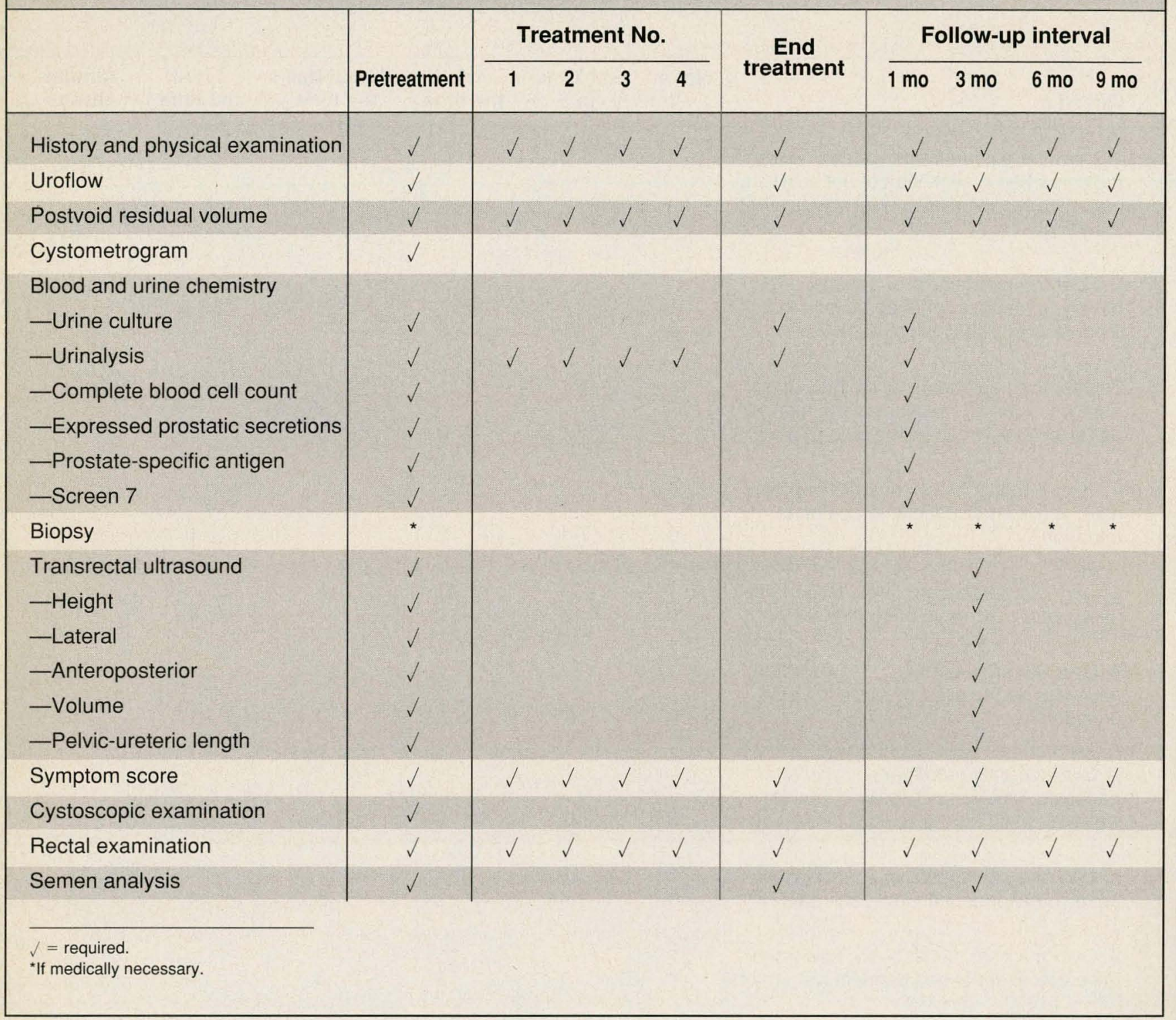

sive to treatment. 5 The diagnosis of chronic nonbacterial prostatitis is one of exclusion. The proper clinical symptoms along with a negative expressed prostatic secretion and negative Chlamydia and Ureaplasma titers provide the diagnosis. Because Chlamydia and Ureaplasma are difficult to obtain in culture media, the urologist often treats for infection with these microorganisms empirically before reaching the diagnosis of chronic nonbacterial prostatitis.

Some investigators believe that chronic nonbacterial prostatitis is not an infec- tious disease. Such factors as prostaglandins, autoimmunity, neuromuscular dysfunction of the bladder neck, and allergy have been suggested, usually with little objective support. In view of the uncertainty about etiology, treatment of nonbacterial prostatitis remains speculative. Most treatment is aimed at ameliorating symptoms; general relaxant measures, warm tub baths, and spasmolytics may be helpful. Nonsteroidal anti-inflammatory agents may be indicated for pain. Although radical prostatectomy will cure chronic prostatitis, the risks and complications are usually excessive in view of the benign nature of the disease. Subtotal prostatectomy via transurethral resection of the prostate, retropubic or suprapubic, has a low cure rate of only $30 \% .8$ Most treatment regimens can be followed up primarily by subjective symptom scores, as no objective parameters have been found to be consistent or reliable.

Transurethral microwave hyperthermia for the treatment of chronic nonbacterial prostatitis has been shown to be safe and effective. ${ }^{2}$ Servadio and Leib ${ }^{5}$ 
had a greater than $70 \%$ response rate in the men they treated. They looked at 45 men with chronic nonbacterial prostatitis and treated them with transrectal hyperthermia one time a week for 6 weeks. They found that $75 \%$ of the men improved with a $45 \%$ to $60 \%$ decrease in symptom scores. Nickel and Sorenson 12 administered one transurethral treatment to 19 patients with chronic nonbacterial prostatitis; $47 \%$ of these patients had a symptom score decrease of greater than $75 \%$ and another $16 \%$ had moderate improvement. Choi and colleagues ${ }^{13}$ reported similar results to those of Nickel and Sorenson. They subjected 61 patients to one transurethral microwave hyperthermia treatment and found $23 \%$ had a complete response, while $43 \%$ had a partial response.

These studies, in addition to our study, show that transurethral microwave hyperthermia is an effective mode of treatment for chronic nonbacterial prostatitis. Earlier studies (Servadio and coworkers 2,5 ) used the transrectal approach whereas the newer studies (those of Nickel 12 and Choi ${ }^{13}$ and their respective coworkers and of our own group) treated via the transurethral approach. In the treatment of benign prostatic hyperplasia, transurethral hyperthermia has been shown to be more effective than transrectal hyperthermia in effecting a symptom score decrease and in long-term outcomes. ${ }^{14}$ In the treatment of chronic nonbacterial prostatitis, the transurethral approach seems to be the superior one.

The earlier studies (Servadio and Leib ${ }^{5}$ ) were of patients who received six treatments (one time a week for 6 weeks), whereas the later studies (those of Nickel's12 and Choi's 13 teams) were of patients treated once. The overall symptom score improvement was similar in these studies. In our study, we treated patients four times (two times a week for 2 weeks) with superior results. Our patients treated on the prescribed treatment protocol had a mean symptom score improvement of $74.9 \%$, which is statistically significant compared with a placebo effect of $30 \%(P<.001)$. Even our single patient treated one time (sim-

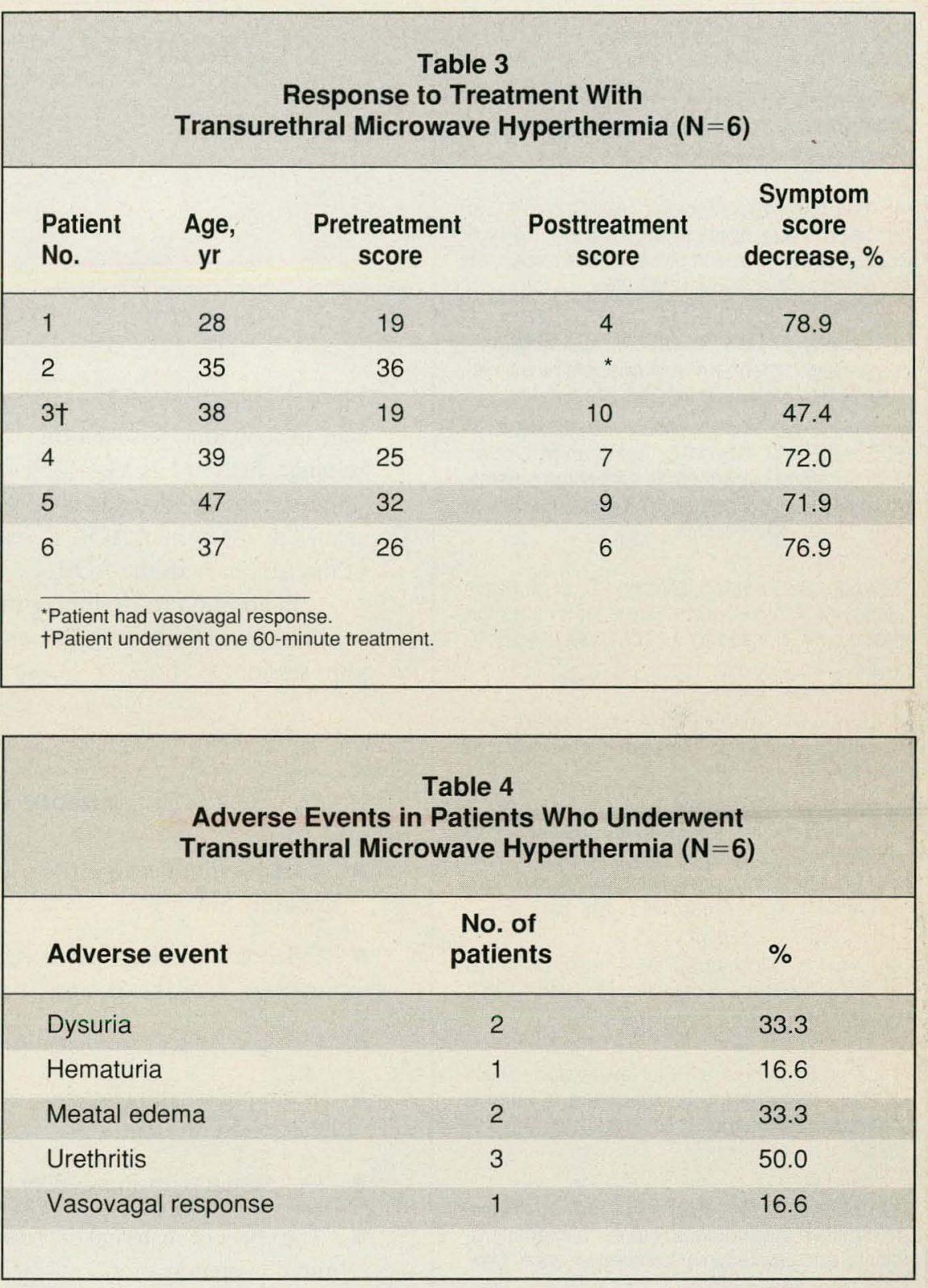

ilar to the protocol of Nickel and Choi and their respective coworkers) had a symptom score decrease of $47.4 \%$. Based on these findings, patients treated a single session had a worse clinical outcome.

\section{Comment}

Based on the results of our study (although limited by size) and a review of the literature, transurethral microwave hyperthermia, using four separate administrations, is a superior protocol for the treatment of chronic nonbacterial prostatitis. This treatment modality in our experience seems to be safe and effective and could be used in patients who have chronic nonbacterial prostatitis unresponsive to medical therapy. Although larger, blinded studies need to be done, transurethral microwave hyperthermia is the only treatment modality with significant documented improvement for patients with chronic nonbacterial prostatitis.

\section{References}

1. Kapoor DA, Reddy PK: Surgical alternatives to TURP in the management of BPH. AUA Update Series 1993;12(3):20.

2. Servadio C, Leib Z, Lev A: Diseases of 
prostate treated by local microwave hyperthermia. Urology 1987;30:97-99.

3. Harada T, Etori K, Kumazaki T, et al: Microwave surgical treatment of diseases of the prostate. Urology 1985;26:572-576.

4. Astrahan MA, Sapozink MD, Cohen D, et al: Microwave application for transurethral hyperthermia of benign prostatic hyperplasia. Int J Hyperthermia 1989;5:283-296.

5. Servadio C, Leib ZC: Chronic abacterial prostatitis and hyperthermia: A possible new treatment? Br J Urol 1991;67:308-311.

6. Sapozink M, Boyd SD, Astrahan MA, et al: Transurethral hyperthermia for benign prostatic hyperplasia: Preliminary clinical results. $J$ Urol 1990;143:944-950.

7. Linder A, Siegel YI, Saranga D, et al: Complications in hyperthermia treatment of benign prostatic hyperplasia. J Urol 1990;144:13901392.

8. Berger R, Hanno PM: A spectrum of prostatitis syndromes. Patient Care May 15, 1990;24:95-111.

9. Crawford ED: Prostatitis: A constellation of disease entities. Urology Grand Rounds. Chicago, III, The Education Division of McCann Healthcare Advertising, 1988, No. 22.

10. Thin R, Simmons PD: Chronic bacterial and non-bacterial prostatitis. Br J Urol 1983; 55:513-518.

11. Thin R, Simmons PD: Review and results of four regimens for the treatment of chronic non-bacterial prostatitis. Br J Urol 1983;55:519521.

12. Nickel J, Sorenson R: Transurethral microwave thermotherapy of nonbacterial prostatitis and prostodynia: Initial experience. Urology 1994;44:458-460.

13. Choi NG, Soh SH, Yoon TH, et al: Clinical experience with transurethral microwave thermotherapy for chronic nonbacterial prostatitis and prostodynia. J Endourol 1994;8:61-64.

14. Stawartz B, Szmiglelski S, Ogrodnik J, et al: A comparison of transurethral and transrectal microwave hyperthermia in poor surgical risk benign prostatic hyperplasia patients. J Urol 1991;146:353-357.

\section{$\triangle C$ Coming in...}

\section{The DO}

The February issue of The DO will offer extensive coverage of the American Osteopathic Association's 101st Annual Convention and Scientific Seminar, held in Las Vegas, Nev, October 7 through 11, 1996. The convention featured educational programs of 13 osteopathic specialty societies as well as the annual AOA Research Conference and the annual meeting of the Auxiliary to the AOA.

In addition to recapping the programs of each of these groups, the February issue will cover the convention's opening session and its annual joint session.

\section{Future issues of $J A O A$}

- "Variables influencing the use of osteopathic manipulative treatment in family practice"

- "Osteopathic manipulative treatment: Student attitudes before and after intensive clinical exposure"

- "Cardiovascular manifestations of Lyme disease"

- "Team physician preferences at National Collegiate Athletic Association Division I universities"

- "Delusional parasitosis: A practical guide to evaluation"

- "The effect of maternal depression on premature infant health during the initial hospitalization"

- "Observatory clues to aid in the diagnosing of diastasis symphysis pubis (An Underreported complication of parturition)"

- "Preliminary results on the effects of manipulative therapy on spasmodic torticollis"

- "Repositioning maneuver for benign paroxysmal positional vertigo"

- "Establishment of behavioral parameters for the evaluation of osteopathic treatment principles in a rat model of arthritis"

- "Martial arts injuries"

- "Diagnosis and treatment of childhood and adolescent depression"

- "Osteopathic considerations of reflex sympathetic dystrophy"

- "Evaluation of a critical pathway for stroke which is initiated in the emergency department" 